Ricardo L. Ramirez Jr., MD

Windolyn D. Panganiban, MD

Joel A. Romualdez, MD

Department of Otorhinolaryngology

Head and Neck Surgery

St. Luke's Medical Center
Correspondence: Ricardo L. Ramirez Jr.,MD

Department of Otorhinolaryngology Head and Neck Surgery

St. Luke's Medical Center

279 E. Rodriguez Ave, Quezon City 1102

Philippines

Telefax: (632) 7230101 local 5543

E-mail: rikrik_2006@yahoo.com

Reprints will not be available from the author.

No funding support was received for this study. The authors signed disclosures that they have no proprietary or financial interest with an organization that may have a direct interest in the subject matter of this manuscript, or in any product used or cited in this study.

Presented at the Descriptive Research Contest (3rd Place) Philippine Society of Otolaryngology-Head and Neck Surgery, Jade Valley Restaurant, Quezon City, Philippines, September 25, 2008.

\section{Subjective and Objective Assessment of Olfactory Function in Post-Laryngectomy Patients}

\begin{abstract}
Objective: To determine the olfactory function among post-laryngectomy patients using a questionnaire adapted from that of the Smell and Taste Clinic of Hospital of University Pennsylvania (HUP) and the Santo Tomas Smell Identification Test.
\end{abstract}

\section{Methods:}

Design: Descriptive study

Setting: Tertiary Private Hospital Outpatient Department

Patients: Twenty five subjects who had undergone total laryngectomy and met inclusion and exclusion criteria underwent rigid nasal endoscopy and olfactory function assessment using an adaptation of the questionnaire of the Smell and Taste Clinic of Hospital of University Pennsylvania (HUP) and the Santo Tomas Smell Identification Test (ST-SIT).

Results: Twenty one male subjects completed olfactory testing. All had subjective sense of smell before laryngectomy. Statistically significant correlation was noted between the subjective postoperative smell function and the objective olfactory function test scores. There was no statistically significant difference noted in the ST SIT scores with regards age, duration from laryngectomy to olfactory testing, number of smoking pack- years, use of olfactory technique/maneuver, loss of appetite and adjunctive chemotherapy and radiotherapy.

Conclusion: All subjects post-laryngectomy had subjective complaints of varying levels of olfactory difficulties based on a structured questionnaire and were documented to be anosmic by an objective smell identification test. Olfactory problems following laryngectomy can have significant effects on the lives of laryngectomees, and health care providers should be knowledgeable of available management options for this condition.

Key words: olfaction, anosmia, total laryngectomy, olfactory testing

Olfactory deterioration affects the quality of life of a person, impairing appreciation of food 


\section{ORIGINAL ARTICLES}

and beverage and depriving environmental cues to warn of potentially life-threatening situations. The perception of taste of chocolate, coffee, tea and meat are dependent on retronasal stimulation of olfactory receptors and the appreciation of such tastes will also be negatively influenced by the loss of the sense of smell. ${ }^{1}$ Total laryngectomy results in a permanent separation between the upper and lower airways with a wide range of adverse effects. The change in anatomy leads to deterioration in pulmonary function as well as loss of the normal senses of smell and taste, loss of voice and associated physical and psychosocial problems. ${ }^{2}$ While some observe that the immediate, inevitable anosmia resulting from laryngectomy does not resolve even after eight years, ${ }^{3}$ others have reported improvement in olfaction within the first 6 months after surgery and documented the presence of a relatively normal sense in some laryngectomees. ${ }^{4}$ Laryngectomees have been divided into 2 groups, smellers and non-smellers, based on odor detection and/or odor differentiation tests. ${ }^{5}$ With the increasing number of post-laryngectomy patients, the lack of resources for olfactory rehabilitation, and the paucity of local literature on the topic, this study aims to subjectively and objectively determine the olfactory function of our subject population.

\section{MATERIALS AND METHODS}

\section{Subjects}

Twenty five subjects who had previously undergone total laryngectomy and were all members of a support group of laryngectomized patients that consulted at the out-patient department of St. Luke's Medical Center met inclusion and exclusion criteria, and informed consent was obtained from each. All patients had a subjective sense of smell before laryngectomy and no history of head trauma, chronic sinusitis, nasal allergies or severe respiratory infection which may cause olfactory deterioration. Rigid nasal endoscopy was performed on all to detect any anatomic abnormalities like presence of nasal deviation, nasal polyposis and nasal discharge that could affect nasal airflow and impair smell.

Patients were excluded if their surgery, chemotherapy and radiotherapy had been performed within less than 3 months. The cut-off point of 3 months was chosen to allow most side effects of the surgery, chemotherapy and radiotherapy to subside and the physical conditions of the patient to stabilize before olfactory testing.

\section{Data Collection}

An adaptation of the questionnaire of the Smell and Taste Clinic of the Hospital of the University of Pennsylvania (HUP) ${ }^{6}$ was administered to each subject to assess their perceived olfactory function. The questionnaire was revised by removing sections on taste symptoms, endocrine information and questions regarding depression on the standard test questionnaire of the Smell and Taste Clinic of the Hospital of the University of Pennsylvania. No local translation or validation of the revised questionnaire was made.

Medical history questions included history of pre and post operative radiotherapy and/or chemotherapy, sinus or nasal problems, serious upper respiratory problems, history of head and neck surgery, smoking history, nasal allergies and family history of smell problems. Smell symptoms items included olfactory disorder prior to laryngectomy, sense of smell immediately after laryngectomy, present sense of olfaction, degree of change in olfaction, technique/maneuver used to improve sense of smell, and the presence of loss of appetite due to the disorder in olfaction. Subjects were asked to rank problems associated with laryngectomy in order of its significance in their everyday life which included pain, smell problems, taste problems, shoulder dysfunction, physical disfigurement, and airway/tracheostomy problems, eating, chewing and swallowing.

Olfactory function of each subject was evaluated using the standardized, locally-validated Santo Tomas Smell Identification Test (ST-SIT). ${ }^{7}$ This test uses 45 odorants, each enclosed in an opaque polyethylene squeeze bottle. Each odorant is smelled by the subject and identified from a written list of choices. The odorants had a corresponding score and the summation of these scores serve to discriminate those with normal olfactory function from those with olfaction problems. This test was able to qualify whether a person is anosmic, hyposmic or has normal olfactory function.

\section{Data Analysis}

Demographic, clinical and smell and nasal characteristics were analyzed as frequencies and percentages. Paired $t$ test was used in comparing smell functions before and after laryngectomy. A two-tailed statistical analysis was done with a $p$ value of $<0.05$ being considered significant. Data analyses were computed using SPSS (Statistical Package for Social Sciences) for Windows version 16.0.

\section{RESULTS}

A total of 21 male subjects with age range of 56 to 76 years were included in the study. Olfactory testing was performed as early as 3 months after the total laryngectomy, chemotherapy and radiotherapy to as late as 14 years after treatment.

Table 1 summarizes the distribution of patients based on history of chemotherapy/radiotherapy, smoking history, degree of smell impairment, degree of change in olfactory function, compensatory techniques and length of time from surgery to the development of smell impairment. 
ORIGINAL ARTICLES

\section{Table 1. Demographic and Clinical Characteristics}

\begin{tabular}{ll} 
Characteristics & (N=21) \\
Gender (Male) & $21(100)$ \\
Age (mean + SD) & $64.95+6.6$ yrs \\
Duration from operation to testing (mean + SD) & $4.48+4.48$ yrs \\
Chemo and Radiotherapy & \\
Pre-op chemo \& radiotherapy & $7(33.3)$ \\
Post-op Chemotherapy & $2(9.5)$ \\
Post op Radiotherapy & $5(23.8)$ \\
Post -op Chemo \& Radiotherapy & $4(19.0)$ \\
No Chemo \& Radiotherapy & $3(14.3)$ \\
History of Sinus / Nasal Problems, Respiratory & $0(0)$ \\
Problems, Previous Head and Neck Operation, Allergies & \\
and exposure to chemicals & \\
Smoking History & $19(90.5)$ \\
Smoking Pack Years & \\
Less than 20 & $2(9.5)$ \\
21 to 40 & $13(61.9)$ \\
41 to 60 & $4(19.0)$ \\
61 to 80 & $2(9.5)$ \\
\hline
\end{tabular}

Table 2. Ranking of problems associated with total laryngectomy *

\begin{tabular}{|c|c|c|}
\hline & Mean & Rank \\
\hline Eating & 2.25 & 1 \\
\hline Speaking & 2.40 & 2 \\
\hline Smell & 2.90 & 3 \\
\hline Airway & 3.00 & 4 \\
\hline Shoulder & 3.14 & 5 \\
\hline Taste & 3.60 & 6 \\
\hline Pain & 3.38 & 6 \\
\hline Physical & 4.15 & 7 \\
\hline
\end{tabular}

${ }^{*}$ ranked by the subjects according to its significance in their daily life

There was no significant correlation between olfactory score and other demographic and clinical characteristics such as length of time post-surgery, age, number of smoking pack years, loss of appetite and interference with daily activities.

Table 2 summaries the different problems associated with total laryngectomy as ranked by the subjects. Eating and swallowing were ranked first followed closely by communication problems. The smell problem was ranked higher than airway/tracheostomy problems. Table 3 presents the olfactory characteristics before and after laryngectomy. Because all subjects claimed no problems prior to laryngectomy, paired $t$-test results yielded $p$ values of $<0.05$ for all characteristics. There was
Table 3. Olfactory characteristics before and after laryngectomy

\begin{tabular}{|c|c|c|}
\hline \multirow[t]{2}{*}{ Characteristics } & \multicolumn{2}{|c|}{ Smelling Problem, n (\%) } \\
\hline & Pre-operative & Post-operative \\
\hline Changes in Smell & $0(0)$ & $21(100)$ \\
\hline Phantosmia & $0(0)$ & $1(4.8)$ \\
\hline Hypersensitive & $0(0)$ & $2(9.5)$ \\
\hline Partial Loss & $0(0)$ & $4(19.0)$ \\
\hline Complete Loss & $0(0)$ & $14(66.7)$ \\
\hline \multicolumn{3}{|l|}{ Degree of Change } \\
\hline Mild & $0(0)$ & $3(14.3)$ \\
\hline Moderate & $0(0)$ & $6(28.6)$ \\
\hline Severe & $0(0)$ & $12(57.1)$ \\
\hline \multicolumn{3}{|l|}{ Smell Condition } \\
\hline Getting better & $0(0)$ & $5(23.8)$ \\
\hline Getting worse & $0(0)$ & $7(33.3)$ \\
\hline Fluctuates & $0(0)$ & $2(9.5)$ \\
\hline No improvement & $0(0)$ & $7(33.3)$ \\
\hline Use of technique or maneuver & $0(0)$ & $2(9.5)$ \\
\hline \multicolumn{3}{|l|}{ Technique or maneuver } \\
\hline Sniffing & $0(0)$ & $1(4.8)$ \\
\hline Swallowing & $0(0)$ & $1(4.8)$ \\
\hline Loss of Appetite & $0(0)$ & $14(66.7)$ \\
\hline Smell interferes with everyday functioning & $0(0)$ & $17(81.0)$ \\
\hline
\end{tabular}

a statistically significant correlation between subjective post-operative smell function and the objective olfactory function test scores. The subjects ST-SIT scores ranged from 20-65 with a mean ST-SIT score of 47.26 , indicating that all had olfactory dysfunction, and that all had anosmia.

\section{DISCUSSION}

This study verified that indeed, a majority of laryngectomized patients have some level of subjective olfactory difficulty brought about by the transformation in upper airway physiology as a result of the permanently-created stoma. All our 21 male patients claimed to have no olfactory deficit prior to total laryngectomy. All claimed to have smell function disabilities post-surgery, confirmed in the course of evaluation. In the structured questionnaire, two-thirds of the subjects complained of complete loss of the sense of smell with more than half 
indicating a severe change in their sensory perception occurring within a month after laryngectomy in almost all patients. Majority of the respondents also felt that their smell problem affected their activities and daily functioning.

Although olfaction was noted to have decreased and remain unimproved among a majority of our patients, two (who had mastered esophageal speech) had consciously or unconsciously acquired techniques to improve their weakened sense of smell. One was observed to swallow in air after sniffing the odorants during the smell identification test. The other was noted to use his facial and nasal muscles excessively during sniffing. Although their olfaction test scores did not reflect any relation to these maneuvers, further tests involving larger samples may corroborate Hilgers ${ }^{8}$ positive correlation between quality of esophageal voice and olfaction acuity. This may reflect better control of oropharyngeal musculature in good esophageal speakers, enabling them to pump air into the nasal cavity retronasally. Some smell specialists have exploited such special maneuvers to help patients recover their sense of smell with varying success. In particular, Hilgers and his team ${ }^{7}$ report excellent long-term success with olfaction rehabilitation using the nasal airflow-inducing maneuver or the "polite yawning technique."

The absence of significant correlation between time in years since laryngectomy and olfactory testing is supported by Leon et al. ${ }^{9}$ who found no significant correlation between time in years since laryngectomy and oronasal or retronasal psychophysical testing scores. This may support the case for earlier olfactory rehabilitation following laryngectomy, rather than the practice of later ("wait and see"), or no intervention.

Variations in the problems associated with laryngectomy that were ranked by the subjects may be due in part to most of our respondents having had laryngectomy several years before this study. While patients with laryngectomies of several months' duration may be more concerned about airway and tracheostomy problems, patients who have had a permanent stoma for several years may be more accustomed and adapted to it that they no longer feel that it is a major cause for concern.

Majority of the respondents developed loss of appetite and claimed that loss of olfaction interfered with everyday functioning in terms of taste perception leading to poor nutrition and in some cases, eventual weight loss. This may be due to laryngectomy disrupting olfaction, both retronasal (odorant molecules from the oral cavity delivered the olfactory cleft via nasopharynx and posterior choanae) and orthonasal (odorant molecules delivered to olfactory epithelium via the nares).

Our study showed that all subjects post-laryngectomy had subjective complaints of varying levels of olfactory difficulties based on a structured questionnaire and were documented to be anosmic by an objective smell identification test. Olfactory problems following laryngectomy can have significant effects on the lives of laryngectomees and health care providers should be knowledgeable of available management options for this condition.

\section{ACKNOWLEDGEMENT}

We thank Mr. Emer L. Roxas and the members of Nu Vois Association of the Philippines and the Philippine Laryngectomee Club for actively participating by providing subjects for this study and Ms. Catherine C. Cristobal of the Clinical Epidemiology Department of St. Luke's Medical Center for help with statistical analysis.

\section{REFERENCES}

1. Burdarch KJ, Doty RL. The effects of mouth movements, swallowing and spitting on retronasal odor perception. Physiol Behav. 1987; 41: 353-356.

2. Risberg-Berlin B, Ylitalo R, Finizia C. Screening and rehabilitation of olfaction after total laryngectomy in Swedish patient: results from intervention study using the nasal airflowinducing maneuver. Arch Otolaryngol Head Neck Surg. 2006; 132 (3): 301-306.

3. Henkin RI, Hoye RC, Ketcham AS, Gould WJ. Hyposmia following laryngectomy. Lancet 1968; 2: 479-481.

4. Moore-Gillon V. The nose after laryngectomy. J R Soc Med. 1985; 78: 435-439.

5. van Dam FSAM, Hilgers FJM, Emsbroek G, Touw FI, van As CJ, De Jong N . Deterioration of olfaction and gestation as a consequence of total laryngectomy. Laryngoscope. 1999; 109: 1150-1155

6. Deems DA, Doty RL, Settle RG, Moore-Gillon V, Shaman P, Mester AF, Kimmelman $C P$, Brightman VJ, Snow JB. Smell and taste disorders: a study of 750 patients from the University of Pennsylvania Smell and Taste Center. Arch Otolaryngol Head Neck Surg 1991; 117: 519-528

7. David J, Campomanes B, Dalupang J, Loberiza F. Smell Identification Test. Philipp J Otolaryngol Head Neck Surg 1994;62-68.

8. Hilgers FJM, van Dam FSAM, Keyzers S, Koster MN, van As C, Muller MJ. Rehabilitation of Olfaction after Laryngectomy by Means of a Nasal Airflow-Inducing Maneuver. Arch Otolaryngol Head and Neck Surg 2000; 126: 726-732.

9. Leon EA, Catalanotto FA, Werning JW. Retronasal and Orthonasal Olfactory Ability after Laryngectomy. Arch Otolaryngol Head Neck Surg 2007;133:32-36.

10. Luessen AW, Kobal G, Wolfensberger M. Assessing Olfactory Function in Laryngectomees using the Sniffin Sticks Test Battery and Chemosensory Evoked Potentials. Laryngoscope 2000; 110:303-307. 